\title{
Hemophilia Gene Therapy: Ready for Prime Time?
}

\author{
Thierry VandenDriessche ${ }^{1,2, *}$ and Marinee K. Chuah ${ }^{1,2, *}$ \\ ${ }^{1}$ Department of Gene Therapy \& Regenerative Medicine, Free University of Brussels (VUB), Brussels, Belgium; ${ }^{2}$ Center for Molecular \& Vascular Biology, Department \\ of Cardiovascular Sciences, University of Leuven, Leuven, Belgium.
}

Hemophilia A and B are congenital, X-linked bleeding disorders caused by mutations in the genes encoding for the blood clotting factor VIII (FVIII) or factor IX (FIX), respectively. Since the beginning of gene therapy, hemophilia has been considered an attractive disease target that served as a trailblazer for the field at large. Different technologies have been explored to efficiently and safely deliver the therapeutic FVIII and FIX genes into the patients' cells. Currently, the most promising vectors for hemophilia gene therapy are adenoassociated viral vectors (AAVs) and lentiviral vectors. More recently, gene editing approaches based on designer nucleases or CRISPR/Cas, have also been considered to minimize risks associated with random vector integration and insertional mutagenesis though off-target issues would have to be carefully and comprehensively assessed. In the past two decades, several phase 1 hemophilia gene therapy clinical trials have been initiated with varying success. In particular, the early gene therapy clinical trials in hemophilia B patients based on AAV showed either transient or subtherapeutic clotting factor expression levels. This could be ascribed, at least in part, to suboptimal vector design and/or inadvertent immune consequences triggering hepatic inflammation. Hence, there was an unmet need to further increase vector safety and efficacy in future trials, preferably by using lower vector doses. It is particularly encouraging that sustained therapeutic FVIII and FIX expression levels have recently been attained after gene therapy in patients with severe hemophilia paving the way towards pivotal trials and commercialization. Nevertheless, transient liver toxicity still occurs and the use of transient immunosuppression was still required to curtail inadvertent immune responses, especially at high vector doses. To further boost clotting factor expression levels, codon-usage optimized synthetic FVIII or FIX transgenes have been employed. Alternatively, we and others have shown that the incorporation of hyperactive gain-of-function R338L mutation in the FIX gene substantially increased the overall efficacy. It is inevitable that the continued improvements in vector engineering and new insights in the vectorpatient interactions will further benefit the development of a safe and effective cure for hemophilia A and B.

Keywords: factor IX, factor VIII, coagulation, hemophilia, AAV, lentiviral, CRISPR, ZFN, factor IX Padua

\section{INTRODUCTION}

HEMOPHILIA A AND B are congenital X-linked bleeding disorders caused by mutations of genes encoding the blood clotting factor VIII (FVIII) or factor IX (FIX), respectively. Consequently, these disorders are characterized by a bleeding disorder due to a deficiency in the corresponding clotting factors. Patients often suffer from recurrent bleeds and chronic joint damage resulting in a crippling arthropathy. The bleeding could even be fatal in cases of intracranial hemorrhage. According to the
World Federation of Hemophilia, the diseases affect an estimated 400,000 individuals worldwide, ${ }^{1}$ of which $80-85 \%$ are affected by hemophilia A and the remaining $15 \%$ by hemophilia B. Current treatment consists of protein substitution therapy (PST) with recombinant or plasma-derived clotting factors. Although PST improved the patient's quality of life and significantly prolonged life expectancy, it has several drawbacks. First, this treatment is noncurative, implying that the patient remains at risk of developing bleeding episodes

${ }^{*}$ Correspondence: Dr. Thierry VandenDriessche or Dr. Marinee Chuah, Department of Gene Therapy \& Regenerative Medicine, Faculty of Medicine and Pharmacy, Free University of Brussels, Building D, Room D306, Laarbeeklaan 103 B-1090,Brussels, Belgium. E-mail: thierry.vandendriessche@vub.ac.be; marinee.chuah@vub.ac.be

(c) Thierry VandenDriessche and Marinee K. Chuah 2017; Published by Mary Ann Liebert, Inc. This is an Open Access article distributed under the terms of the Creative Commons Attribution License, which permits unrestricted use, distribution, and reproduction in any medium, provided the original work is properly cited. 
and/or chronic joint damage. The second constraint pertains to the clotting factors' short half-lives, requiring frequent treatment with prophylaxis or on demand using relatively large doses of FVIII or FIX. Finally, some patients develop neutralizing antibodies (clinically defined as inhibitors) against the injected FVIII or FIX proteins that render further PST ineffective. Several next-generation proteinbased therapeutics have recently been developed that prolong the half-life of the clotting factors, for instance by pegylation or fusion with albumin and immunoglobulin domains. ${ }^{2}$ Consequently, these second-generation products require less frequent administration and represent a new benchmark for all emerging products to treat hemophilia patients, including gene therapy. Given the intrinsic limitations of PST, there is still a need to develop a long-term cure for hemophilia via gene therapy.

The current review celebrates the 25th anniversary of the European Society of Gene and Cell Therapy. Our own personal journey in the field of gene therapy for hemophilia coincidentally also started exactly 25 years ago. This allowed us to witness firsthand the exciting developments in gene therapy for hemophilia that had an impact on so many other diseases since then. In this review, we will highlight the most relevant and most recent developments in the field of gene therapy for hemophilia A and B. We will also discuss some of the outstanding questions and remaining challenges that would still need to be addressed. It is beyond the scope of the current review to provide a comprehensive description of all of the possible strategies and animal studies that have been published relating to hemophilia gene therapy in the past 30 years, which have been covered by other earlier reviews on the subject. ${ }^{3-5}$ Instead, we will focus on highlighting a few key concepts with specific emphasis clinical translation.

\section{GENE THERAPY}

The main objective of developing gene therapy for hemophilia is to achieve prolonged high-level expression of clotting factors and stably correct the bleeding disease. Essentially, there are two distinct approaches to achieve this objective. The first approach is FVIII or FIX gene delivery to long-lived post mitotic cells, such as hepatocytes or skeletal muscle cells. In this case, the therapeutic genes should either integrate into the target cell genome or persist as episomal DNA. Alternatively, FVIII or FIX genes could be delivered to stem/progenitor cells, such as hematopoietic stem cells (HSCs), using integrating (viral or nonviral) vectors in order to achieve long-lasting expression through their progeny. Designing the optimal FVIII and FIX expression cassette and delivering these constructs into the appropriate target cells in a safe and efficient manner will be essential toward achieving a cure for hemophilia. Ideally, gene therapy should not result in the induction of inhibitors or should enable clearance of pre-existing inhibitors through induction of FVIII- or FIXspecific immune tolerance, which has been accomplished in preclinical studies. ${ }^{6,7}$ The ability to induce immune tolerance to FVIII or FIX after gene therapy varies depending on several confounding variables, including the transgene product, the vector design, the target cells, and the underlying mutation of the endogenous FVIII or FIX gene.

\section{Designing optimal $F V I I I$ and $F I X$ expression cassettes}

As with any gene therapy approach, it is critically important to express high levels of the therapeutic protein using the lowest possible vector dose to minimize untoward vector-associated immune responses or toxicity. This has been particularly challenging since there are several bottlenecks at the transcriptional, translational and post-translational level, especially for FVIII. ${ }^{8}$ It is therefore necessary to overcome each of these limiting steps using rationally designed transgenes and expression constructs. Incremental improvements at the transcriptional, translational, and/or post-translational levels may ultimately translate into significant dose benefits in clinical trials in hemophilia patients. All of the gene therapy approaches rely on the use of FVIII and FIX cDNAs instead of the full-length transgenes per se. Nevertheless, in many cases, introns were incorporated in the constructs ${ }^{9,10}$ to further boost expression levels that act possibly, at least in part, by facilitating extra-nuclear transport of the cognate transcripts.

The endogenous FVIII and FIX promoters are not particularly robust and are therefore not ideally suited for gene therapy for hemophilia. Instead, to boost the FVIII and FIX mRNA levels, we and others have explored the use of robust tissue-specific heterologous promoters, such as $\alpha$ 1-antitrypsin or transthyrethin. ${ }^{10-12}$ These promoters could be combined with other regulatory elements (e.g., HCR, enhancers) to further boost expression. ${ }^{11}$ More recently, we have developed a novel approach to identify robust human hepatocytespecific cis-regulatory modules (CRMs) using genome-wide computational strategies instead of the conventional trial-and-error approaches. The use of these CRMs, in conjunction with existing promoters, resulted in relatively robust increases in FIX or 
F'VIII gene expression when used in the context of different gene therapy vectors. ${ }^{13-15}$ It is reassuring that these CRMs increased expression of heterologous transgenes, including FVIII and FIX, without increasing the risk of insertional oncogenesis of integrating vectors, even in highly sensitive tumor-prone mouse models. ${ }^{15}$ Alternatively, synthetic transcriptional enhancers were generated de novo by random ligation of synthetic oligonucleotides coding for binding sites of hepatic transcription factors and in vitro screening in hepatic cell lines. ${ }^{16}$ The specificity of the promoter and regulatory elements can have important consequences for the immune response directed against the transgene products ${ }^{16,17}$ (see below).

More than 20 years ago, we pioneered the use of codon-optimization of clotting factor genes in attempt to increase their expression. ${ }^{9}$ Since then, we and others have continued to develop codonoptimized FVIII and FIX transgenes ${ }^{10,13,18,19}$ that resulted in higher levels of protein expression for the same amount of mRNA. One of the main advantages of codon optimization is that it does not alter any of the amino acids encoded by the $F V I I I$ or FIX transgene obviating possible immunogenicity concerns. Typically, codon-usage optimization resulted in a 3-fold increase in FIX protein expression levels, but in the case of FVIII an unexpectedly greater impact on FVIII protein levels was apparent (up to 44 -fold). ${ }^{18}$ Deletion of the B-domain $(F V I I I \Delta B)$ was also shown to be essential in boosting FVIII expression levels, by increasing the FVIII mRNA levels compared with the full-length FVIII cDNA. ${ }^{20}$ Nevertheless, deletion of the entire B-domain compromises specific post-translational intracellular FVIII trafficking due to the loss of critical glycosylated residues. ${ }^{21}$ To compensate for this defect, a synthetic B-domain-derived 17amino acid polypeptide was reintroduced in the $F$ VIII $\triangle B$ cDNA that presumably reconstitutes these asparagine-linked oligosaccharides, consistent with a $\sim 3$-fold increase in the secreted FVIII protein levels. ${ }^{19}$ Alternatively, incorporation of a furin cleavage site within the B domain (position $\mathrm{R} 1645 \mathrm{H}$ ) that mimics the canine sequence (histidinehistidine-glutamine-arginine $[\mathrm{HHQR}]$ vs. human arginine-histidine-glutamine-arginine [RHQR]) increased FVIII expression $\sim 2$-fold in hemophilia A mice. ${ }^{22}$ FVIII expression levels could also be increased by generating porcine-human hybrid $F$ VIII $\Delta B$ transgenes. ${ }^{23}$ It has been shown that overexpression of FVIII above $200 \%$ may provoke cellular stress which may in turn increase the risk of inhibitors. ${ }^{24}$ It may therefore be prudent to restrict FVIII expression levels to minimize the risk of such untoward cellular stress responses. However, it is reassuring that FVIII levels up to 75 -fold higher than physiologic levels did not result in detectable transaminitis or liver toxicity. ${ }^{25}$

As an alternative to codon optimization, we showed, for the first time, with liver-directed gene delivery that a relatively robust increase in FIX activity (by up to 8-fold) could be obtained by incorporating a single R338L gain-of-function point mutation in the FIX gene. ${ }^{13,26}$ This point mutation (designated as FIX-Padua) was initially discovered in thrombophilic patients ${ }^{27}$ and this could be harnessed to significantly augment the efficacy of gene therapy for hemophilia B with minimal sequence perturbation. The functional enhancement of this specific point mutation was confirmed in subsequent studies. ${ }^{28-30}$ Several other mutants have been explored that enhance FIX activity. ${ }^{31}$

\section{Target cells for hemophilia gene therapy}

Different cell types have been explored for gene therapy of hemophilia and have been extensively reviewed elsewhere. ${ }^{4,5}$ Some of the most relevant cell types are discussed in more detail below.

The liver is an attractive target organ for both hemophilia A and B gene therapy since FVIII and FIX are naturally produced by liver sinusoidal endothelial cells (LSECs) and hepatocytes, respectively. Consequently, LSECs and hepatocytes have been targeted by different viral and nonviral vector $^{32}$ systems for gene therapy. Perhaps most importantly, the hepatic niche may favor the induction of immune tolerance towards the transgene product, ${ }^{33}$ which may depend, at least in part, on the induction of regulatory T-cells. ${ }^{34,35}$

Skeletal muscle cells have a relatively robust secretory capacity and are equipped with the necessary cellular machinery enabling the post-translational modifications required to generate functional FIX. Though FIX could be secreted into the circulation when expressed in the muscle, off-loading FVIII in the circulation is impaired compared with the liver. This may be due to the relatively large size of FVIII particularly when complexed with vWF. Unlike liverdirected gene transfer, it would appear that muscledirected approaches appear to have a higher risk of inducing immune response against the transgene product. $^{36}$ Despite these limitations, early clinical trials showed that muscle can give rise to sustained FIX expression in patients up to 10 years after gene therapy. ${ }^{37}$

Hematopoietic stem cells (HSCs) have also been explored as targets for hemophilia gene therapy to specifically express FVIII or FIX in different hematopoietic lineages. In particular, HSC-derived erythrocytes, megakaryocytes and their platelet 
progeny could serve as a delivery platform to secrete the clotting factors directly in the circulation following HSC-targeted gene transfer. ${ }^{38}$

\section{Preclinical and clinical studies}

Gene therapy for hemophilia A and B requires an efficient and innocuous gene transfer system that should preferably give rise to sustained, lifelong therapeutic FVIII or FIX expression without any immune or toxic side effects. Several vectors have been developed that are well suited for gene therapy of hemophilia, each with their own advantages and limitations.

\section{Nonviral vectors}

Nonviral vectors typically rely on DNA-based physical or chemical transfection methods. Though these methods typically result in no adaptive immune responses, as opposed to when viral vectors are employed, it is well established that DNA can evoke innate immune responses in vivo, typically involving Toll-like receptor signaling pathways. The gene transfer efficiencies are generally lower compared with most viral vector-mediated gene transfer approaches. Moreover, nonviral transfection of $F V I I I$ or $F I X$-containing constructs typically results in short-term expression of the transgene product, unless selection is applied on ex vivo transfected cells. ${ }^{39}$ A phase 1 clinical trial for hemophilia A had previously been conducted with stably transfected autologous fibroblasts that were electroporated with FVIII-expressing plasmids. After selective expansion, the transfected fibroblasts were implanted into the patient's omentum. Though no adverse events were noted, the therapeutic efficacy was modest and no sustained FVIII expression could be attained. ${ }^{40}$ We and others have shown that the efficiency of stable genomic integration and FIX or FVIII expression levels could be substantially increased using transposons or integrases, consistent with sustained phenotypic correction of the bleeding phenotype in hemophilic mice. ${ }^{15,41-43}$

\section{Gamma-retroviral and lentiviral vectors}

Gamma-retroviral vectors integrate stably into the chromosomes of actively dividing cells. In 1999, we had established the first proof-of-concept that hemophilia A could be cured by gene therapy in a preclinical model of hemophilia $\mathrm{A}^{44}$ To achieve this, neonatal FVIII-deficient mice were used since they are permissive for $\gamma$-retroviral transduction by virtue of the rapid hepatocyte turn-over. This was subsequently confirmed in neonatal hemophilia A and $\mathrm{B}$ dogs. ${ }^{45,46} \mathrm{~A}$ phase 1 clinical trial was conducted in adult hemophilia A patients but FVIII levels were low due to the requirement for cell division. ${ }^{47,48}$ In contrast, lentiviral vectors could transduce quiescent noncycling hepatocytes, leading to relatively efficient transduction in adult livers (reviewed in Matrai et al. ${ }^{49,50}$ ). We demonstrated that lentiviral vectors could also transduce professional antigen-presenting cells (APCs) (i.e., Kupffer cells) and LSECs. ${ }^{51}$ Inadvertent expression of FVIII or FIX in APCs increases the risk of developing inhibitory antibodies that preclude their long-term expression and renders gene therapy ineffective. ${ }^{52,53}$ Recent studies revealed that residual FIX expression in $\mathrm{CD}_{11} \mathrm{~b}^{+}$plasmacytoid dendritic cells contributes to this immune response, whereas FIX expression in conventional dendritic cells or LSECs contributes to immune tolerance instead. ${ }^{54}$ The mechanism of immune tolerance induction following hepatic FVIII or FIX delivery with lentiviral vectors requires induction of regulatory $\mathrm{T}$ cells. ${ }^{17,34}$ Long-term FIX expression could be achieved by preventing expression in APCs through the use of hepatocyte-specific promoters. ${ }^{52,53}$ In some cases, fine-tuning the specificity of expression using microRNA-regulated expression cassettes was required to achieve long-term expression and immune tolerance. ${ }^{16,26,54,55}$ We have shown that FIX activity could be further increased by using a codon-optimized hyperactive FIX-R338L (Padua) after transduction in hemophilic mice with integration-competent or integration-defective lentiviral vectors. ${ }^{26}$ Moreover, sustained therapeutic FIX expression levels could be attained after liverdirected lentiviral transduction in hemophilic dogs, ${ }^{56}$ paving the way toward eventual clinical translation. One advantage of lentiviral vectors is the absence of pre-existing, vector-specific immunity since most individuals have not been naturally pre-exposed to the lentiviral vector components, in contrast to adeno-associated viral (AAV) vectors (see below). Nevertheless, by virtue of their ability to efficiently interact with professional APCs, lentiviral vectors are capable of inducing innate immune responses, consistent with increased production of pro-inflammatory cytokines. ${ }^{53}$

Gamma-retroviral and lentiviral vectors have been employed to deliver FVIII or FIX genes to HSCs and other stem/progenitor cells populations (reviewed in previous publications by Matri et $a l .^{49,50}$ and Vandendriessche et $a l .^{57}$ ). Transplantation of HSC transduced with FVIII or FIX $\gamma$-retroviral or lentiviral in myeloablated recipient mice resulted in phenotypic correction in hemophilic mice and induction of immunological tolerance to the transgene products. One of the attractive features of lentiviral transduction of HSCs is that 
FVIII and FIX expression can be specifically directed to platelets resulting in hemostatic correction in both mouse and dog models of hemophilia. ${ }^{38,58,59}$ As activated blood platelets mediate the primary response to vascular injury, storage of FVIII within platelets may provide a locally inducible treatment to maintain hemostasis for hemophilia A. Remarkably, phenotypic correction of the bleeding disease in hemophilia A mice could even be achieved in the presence of high-titer inhibitory antibodies after lentiviral platelet-directed FVIII expression, which requires von Willebrand factor. ${ }^{57,60,61}$ This strategy could be especially beneficial to treat hemophilia patients with pre-existing inhibitors.

To facilitate HSC engraftment and create a "niche" in the bone marrow, it is necessary to use busulfan or other cytotoxic preconditioning regimens. However, since preconditioning is not without side effects, the risk/benefit ratio would need to be carefully assessed in the context of HSC-based hemophilia gene therapy. To obviate the need for myeloablative preconditioning, direct intraosseous delivery of lentiviral-FVIII vectors has been explored. This resulted in bone marrow and HSC cell transduction in situ and subsequent FVIII production in HSC-derived platelets. ${ }^{62}$ Alternatively, $B$ cells could be employed, since they yielded therapeutic FIX levels in vivo in xeno-transplantated immunodeficient mice without preconditioning. ${ }^{63}$

One of the main safety concerns related to $\gamma$ retroviral and lentiviral vectors relates to the risk of insertional oncogenesis resulting from random genomic integration. ${ }^{50}$ The vector design or the presence of transcriptionally active long terminal repeat can influence this genotoxic risk. Removal of approximately $400 \mathrm{bp}$ in the long terminal repeat region to abolish its transcriptional activity (i.e., self-inactivating vector design) coupled with the use of a promoter in an internal position substantially lowers the risk of insertional oncogenesis. The relative safety of these self-inactivating vectors has been confirmed experimentally in sensitive tumor-prone mouse models using lentivirally transduced HSCs or hepatocytes. ${ }^{56,64}$ This implies that the vector design itself can dramatically decrease the risk of insertional oncogenesis even in tumor-prone mouse models that overestimate this risk. Integration-defective lentiviral vectors that harbor an inactivating mutation in the integrase further minimize the risk of insertional oncogenesis. We showed that transgene expression levels were reduced in comparison with conventional integrase-competent lentiviral vectors. ${ }^{55}$ Nevertheless, relatively robust FIX activity levels could be obtained with integration-defective lentiviral vectors when the FIX-R338L Padua variant was employed. ${ }^{26}$

\section{Adenoviral vectors}

Adenoviral vectors have been extensively studied for hemophilia gene therapy but were largely abandoned after the failed adenoviral vector trial to treat ornithine transcarbamylase deficiency in the late 1990s. Their main disadvantage pertains to the risk of uncontrollable inflammatory reactions following systemic administration that can potentially be fatal. Nevertheless, we and others have shown that high-capacity adenoviral vectors devoid of any viral genes and encoding FVIII or FIX gave rise to robust supraphysiologic clotting factor expression levels, with limited toxicity and no evidence of inflammation in hemophilic mouse and dog models. ${ }^{25,65,66}$ A phase 1 clinical trial was conducted in severe hemophilia A patients, based on the systemic administration of a high-capacity adenoviral vector encoding FVIII. Although very low FVIII levels (approximately 1\%) may have been obtained, the trial was stopped when a transient inflammatory response with hematologic and liver abnormalities became apparent after the first patient was treated. Though the latest-version high-capacity adenoviral vectors result in reduced adaptive immune responses and improved stability of transgene expression, the innate immune response in human subjects is difficult to predict and can still be a serious issue. Consequently, it may not be straightforward to simulate the adenoviral vector-induced innate immune response in preclinical animal models. Current efforts are aimed at minimizing the interaction between highcapacity adenoviral vectors with the innate immune system (e.g., by localized delivery ${ }^{67}$ ) before contemplating future clinical trials with this type of vector for hemophilia gene therapy. However, this will be a tough call given the promising developments with other safer vector systems, particularly AAVs.

\section{Adeno-associated viral vectors}

Hemophilia B. AAV2 vectors can transduce a broad variety of tissues, including muscle and liver. Muscle-directed gene transfer in a mouse or canine hemophilia B model with a missense mutation resulted in stable FIX expression. ${ }^{68,69}$ However, in dogs with a FIX null mutation, high-titer inhibitors could be induced curtailing phenotypic correction. ${ }^{70}$ The overall therapeutic efficiency was increased up to 10 -fold by delivering the AAV-FIX vectors to the muscle intravascularly, under transient immunosuppression. ${ }^{71}$ The risk of inhibitor 
formation was determined by the underlying mutation in the FIX gene, vector dose, and local FIX antigen doses in the transduced muscle and could be prevented by transient immune suppression or by limiting the vector dose per site (reviewed in Wang et $a l .{ }^{72}$ ). Based on these preclinical studies, a phase 1 clinical trial was initiated whereby severe hemophilia B patients with a missense FIX mutation were injected at multiple intramuscular sites with AAV-FIX vectors. ${ }^{73}$ Though circulating FIX levels were low or subtherapeutic, FIX expression was detectable locally in the muscle for at least 10 years with no evidence of anti-FIX antibodies. ${ }^{37}$

The need for repeated muscle injections, and the increased inhibitor risk, justified exploring AAVmediated hepatic gene transfer as an alternative. Initially AAV2 was used, followed by other naturally occurring serotypes (i.e., AAV8, AAV9, AAV5) or capsid variants obtained by evolution and selection ${ }^{74,75}$ or containing specific point mutations. ${ }^{76}$ Preclinical studies with the AAV vectors in murine and canine models of hemophilia or in nonhuman primates have demonstrated persistent therapeutic FIX expression, leading to partial or complete correction of the bleeding phenotype. ${ }^{10,12,53,77-82}$ Long-term correction of the bleeding disease could be achieved after liver-directed AAV2-FIX gene therapy even in inhibitor-prone hemophilia B dogs harboring a $F I X$ null mutation. ${ }^{80}$ It would appear, therefore, that the risk of inhibitor development is reduced after liver-directed gene therapy compared with muscle-targeted therapy. This may possibly be due to the induction of regulatory T cells. ${ }^{33,83}$

These liver-directed preclinical gene therapy studies justified the use of AAV vectors in a phase 1 clinical trial in patients suffering from severe hemophilia B. It was particularly encouraging that therapeutic FIX levels, reaching nearly $12 \%$ of normal levels, could be achieved following liverdirected delivery of AAV-FIX ${ }^{84}$ However, FIX expression was transient and resulted in vector dosedependent hepatotoxicity consistent with plasma elevation of transaminases. One possible hypothesis (designated hereafter as the "T-cell hypothesis") that could account for this observation is that the transduced hepatocytes were able to present AAV capsid-derived antigens in association with major histocompatibility complex class 1 to T cells, which would explain the enzymed-linked immunoSpot (ELISPOT) results from patient peripheral mononuclear blood cells (PMBCs). ${ }^{84,85}$ Although antigen presentation was modest, it may have been sufficient to flag the transduced hepatocytes for - cellmediated destruction. Though attempts were made to simulate this AAV-specific T-cell response in various model systems in vitro and in vivo, ${ }^{86,87}$ it remains somewhat puzzling why this has not been observed in more conventional preclinical disease models (mouse, dogs) or in nonhuman primates.

In a second liver-directed gene therapy trial, severe hemophilia B patients were injected intravenously with an AAV8 vector expressing a codonoptimized FIX from a liver-specific promoter. ${ }^{57,88}$ This trial was based, at least in part, on the rationale that AAV8 allows for a substantial increase in hepatic transduction compared with other serotypes, ${ }^{89,90}$ though this may not necessarily translate to higher species, including humans. This AAV8 serotype exhibits reduced cross-reactivity with preexisting anti-AAV2 antibodies. Interestingly, its uptake by dendritic cells may be reduced compared with conventional AAV2 vectors, potentially resulting in reduced T-cell activation based on preclinical studies. ${ }^{91}$ The AAV vector used in this trial was based on a so-called self-complementary design, ${ }^{82}$ which was believed to overcome one of the limiting steps in AAV transduction, namely the singlestranded DNA to double-stranded transcriptioncompetent double-stranded DNA conversion. ${ }^{92}$ Nevertheless, its effect on expression may vary depending on the expression cassette. The treated subjects expressed FIX above the therapeutic $1 \%$ threshold for several years after vector administration, yielding sustained expression levels ranging between $1 \%$ and $6 \%$ of normal levels over a median period of 3.2 years. This demonstrated, for the first time, that sustained therapeutic FIX levels could be achieved after gene therapy in hemophilia $\mathrm{B}$ patients. In the high-dose group $\left(2 \times 10^{12} \mathrm{vg} / \mathrm{kg}\right)$, a consistent increase in the FIX level to $\sim 5 \%$ was observed in all 6 patients, which resulted in a reduction of more than $90 \%$ in both bleeding episodes and the use of prophylactic FIX concentrate. In this trial, AAV-specific T-cell responses could be detected, but not in all of the patients.

A recent trial based on the same vector design (UniQure; NCT02396342) but using another capsid (AAV5) essentially confirmed these results. In those patients $(n=5)$ who received the initial dose level $\left(5 \times 10^{12} \mathrm{vg} / \mathrm{kg}\right)$ of the AMT-060 vector, an average of $\sim 4 \%$ FIX was attained. In the higherdose cohort $\left(2 \times 10^{13} \mathrm{vg} / \mathrm{kg}\right)(n=5)$, FIX expression increased to almost $7 \%$. This was consistent with a reduced spontaneous bleeding rate and FIX usage to the extent that eight out of nine participants who were on FIX prophylaxis initially did not require prophylaxis any longer. In this trial, AAV-specific T-cell responses could not be detected.

Based on these trial results no apparent benefit of using AAV5 over AAV8 was apparent in terms of 
efficacy. Nevertheless, it is challenging to directly compare these trail results since there were no head-to-head comparisons and there may be many confounding variables that impact on the trial outcome, besides the capsid itself (i.e., ratio of functional to total vector particles, differences in manufacturing and vector purity, patientdependent characteristics, etc.). Strikingly, in both these AAV8 and AAV5 trials, elevated transaminase levels were apparent, consistent with the result obtained in the initial AAV2 trial. ${ }^{84}$ Patients were therefore treated by transient immune suppression with tapering doses of glucocorticoids in the hope of blocking any inadvertent immune responses. Though there is circumstantial evidence supporting the "T-cell hypothesis," some patients in the AAV8 trial had no elevated liver enzyme levels despite an increased in AAV capsid-specific T-cell response. Other patients in both the AAV8 or AAV5 trial had an increase in liver enzyme levels, without any supporting evidence of an AAV capsidspecific T-cell response. This suggests detecting vector-specific T-cells in the peripheral blood may not necessarily be associated with the immune rejection of AAV-transduced hepatocytes, perhaps due to the impact of the liver microenvironment on the local T-cell response. Alternatively, the liver enzyme elevation in some of the subjects may have been unrelated to an AAV- specific T-cell response or the T-cell response may not have been sufficiently robust to clear the transduced hepatocytes. The availability of new mouse models that mimic these responses may ultimately shed light on these controversies. ${ }^{87}$

Based on these AAV2, AAV8, and AAV5 trials, there is a need to further improve the overall efficacy to the extent that lower and safer effective vector doses could be used that, ideally, would not result in transaminitis. We had initially demonstrated that liver-directed gene transfer of a codonusage optimized, hyperfunctional FIX R338L Padua variant resulted in a significant dose advantage without any apparent increased thrombotic or immunogenic risk compared with wild-type FIX. ${ }^{13,26}$ This was independently confirmed in other studies, including canine models. ${ }^{29,30}$ Consequently, this justified the use of a hyperfunctional FIX-R338L Padua variant to treat hemophilia B patients by gene therapy.

It is particularly encouraging that the superiority of the FIX-R338L Padua was recently supported by two independent clinical trials. In the first trial (Shire, NCT01687608), patients were treated with a scAAV8 vector encoding a codonoptimized FIX-R338L Padua variant (BAX335).
Seven patients were treated in three dosing cohorts, and 2 patients had transient FIX activity $>50 \%$. FIX levels persisted in 1 patient from the medium dose cohort of $1 \times 10^{12} \mathrm{vg} / \mathrm{kg}$. None of the patients developed FIX inhibitors. In a second trial (Spark Therapeutics/Pfizer), a more consistent response among the trial subjects was apparent. The single-stranded AAV vector genome in the $S P K$ 9001 vector (NCT02484092) contained a codonoptimized FIX-R338L Padua variant and was packaged using an alternative mutated capsid mutant. The mean steady-state FIX activity level for the 10 participants, 12 weeks treated at the initial dose level $\left(5 \times 10^{11} \mathrm{vg} / \mathrm{kg}\right)$, was sustained at $33 \%$ with no evidence of inhibitory antibodies. Annualized bleeding rate among the 10 patients was reduced by $96 \%$ to a mean of 0.39 annual bleeds, compared with 9.2 bleeds before SPK-9001 administration. In addition, annualized infusion rates were reduced $99 \%$ to a mean of 0.98 annual infusions, compared with 68.5 infusions before SPK-9001 administration. FIX prophylaxis was discontinued. However, 2 patients who showed a transient asymptomatic elevation in liver enzymes and drop in FIX activity, potentially indicating an immune response, were treated with oral corticosteroids.

Hemophilia A. The packaging constraints of AAV initially hampered vector production for hemophilia A gene therapy due to the relatively large size of the FVIII transgene. Typically, relatively high vector doses were required and it remained challenging to accommodate the FVIII transgene with a potent hepatocyte-specific promoter in a single vector. The use of single-chain was preferred over dual-chain vector expressing the heavy and light chain separately. ${ }^{93-96}$ The efficacy of gene therapy for hemophilia A using AAV vectors could be enhanced by using small regulatory elements to drive expression of a B-domain-deleted form of $F V I I I$ by using codon-optimized FVIII cDNA and/or incorporating specific mutations in the FVIII cDNA. ${ }^{19,22,96-99}$ Hemophilia A dogs that received AAV2-cFVIII, AAV6-cFVIII, and AAV8-cFVIII persistently expressed therapeutic levels of FVIII, without antibody formation or other toxicities, for more than 3 years. However, in contrast to mice, liver transduction efficiencies are similar between AAV2, AAV6, and AAV8 serotypes in hemophilia A dogs.

In a more recent study, an AAV8 vector was constructed that contained a $5.2 \mathrm{~kb}$ vector genome encoding a codon-optimized $F$ VIII $\triangle B$ cDNA. A 17-amino-acid peptide (V3) containing glycosylation residues was incorporated in the FVIII $\Delta B$ 
sequence that typically boosted FVIII expression $\sim 3$-fold. Supraphysiologic hFVIII expression levels $(732 \pm 162 \%$ of normal) could be attained in hemophilia A mice that were injected with $2 \times 10^{12} \mathrm{vg} /$ $\mathrm{kg}$. Stable hFVIII expression at $15 \pm 4 \%$ of normal was observed at this dose in a nonhuman primate receiving the lowest vector dose $\left(2 \times 10^{12} \mathrm{vg} / \mathrm{kg}\right)$. However, human FVIII expression above $100 \%$ was observed in three macaques that received a higher dose resulting in neutralizing anti-FVIII antibodies that were abrogated with transient immunosuppression. Whether this V3 element will increase the risk of inhibitors in patients with hemophilia A remains to be seen.

A phase 1/2 clinical trial was recently initated (BioMarin, NCT02576795) based on an AAV5 vector (designated as BMN270) that expressed a B-domain deleted F'VIII (identical to Refacto ${ }^{\mathrm{TM}}$ ). A total of 9 patients have received a single dose of the the BMN270 vector, and 7 of those have been treated at the highest dose. Out of 9 patients, 6 patients who received the highest dose of $6 \times 10^{13}$ $\mathrm{vg} / \mathrm{kg}$ attained FVIII levels above $50 \%$, and one had FVIII levels above $10 \%$, after a follow-up of 16 weeks. The therapeutic outcomes improved over time, with 4 patients reaching 146\% FVIII level by week 20. For the 6 patients at the high dose and previously on a FVIII prophylactic treatment regimen, the mean annualized bleeding rate dropped $91 \%$ from 16.3 before receiving BMN 270 to 1.5 two weeks after receiving the therapy. For those same 6 patients, the mean annualized FVIII infusions fell $98 \%$ from 136.7 to 2.9. Nevertheless, those patients receiving high doses of vectors required a steroid regimen for up to 2 weeks after the gene therapy, whereas the maximum transaminase levels were about twice the upper limit of normal at about 12 weeks after the gene delivery, which then declined over the next few weeks. The vector doses used in this trial were substantially higher than what had been used in the AAV-based hemophilia trials. This poses significant vector manufacturing challenges and may have accounted for the unusual transaminase kinetics.

\section{CONCLUSIONS AND PERSPECTIVES}

Is gene therapy for hemophilia now ready for prime time? This is the "million-dollar question"literally and figuratively, given the high cost of current treatment (PST costs an estimated average $\$ 300,000 / y e a r$ per patient). The prospect for a real cure for hemophilia patients has never looked more promising than it does today. This was made possible through a concerted effort of many investi- gators that contributed to improved vector development and design, vector manufacturing, basic studies in coagulation, immunology and vector-host interactions at the molecular and cellular level, and proof-of-concept studies in preclinical disease models. This required an unprecedented multidisciplinary effort involving basic scientists, clinicians, regulators, and stakeholders from industry that fueled the gene therapy pipeline. The advances in the clinic progressed through several logical stages starting with the demonstration that transient therapeutic clotting factor levels could be attained, to more sustained and higher FVIII and FIX expression levels and activities, reducing bleeding frequency and clotting factor usage, even obviating the need for prophylaxis. In particular, the use of bioengineered clotting factors (i.e., based on codon-optimized FVIII or FIX and/or hyperfunctional FIX variants) and improved vector designs was important to bring gene therapy for hemophilia to the next level. Nevertheless, there are still some issues that will need to be addressed. Traumatic bleeds still occur in patients, and the issue of vector-induced liver inflammation will need to be better understood and overcome. Moreover, the potential risk of insertional oncogenesis continues to raise controversy. ${ }^{100-107}$ Nevertheless, the emergence of efficient site-specific integrating vectors based on designer zinc finger nucleases, CRISPR/ Cas9, or nuclease-free targeting approaches into "safe harbor" loci opens new perspectives to further minimize this risk. ${ }^{108-111,112}$ The careful selection of potent cis-regulatory elements that boost $F I X$ or FVIII expression without any apparent increased risk of insertional oncogenesis is warranted. ${ }^{15}$

An adequate balance between efficacy and safety will need to be continuously assessed in the face of new insights in this rapidly developing field, particularly if the number of patients are expected to substantially increase in the coming years as gene therapy trials move into phases 3 and 4 . Eventual marketing authorization approval by regulatory authorities depends on the successful completion of these larger trials. Only then will gene therapy for hemophilia make a concrete difference in the lives of patients and their families that are affected by these diseases.

\section{ACKNOWLEDGMENTS}

We thank the members of the Department of Gene Therapy and Regenerative Medicine and all of our collaborators for their various contributions to portions of the work presented in this review. We also wish to thank Fonds voor Wetenschappelijk Onderzoek (FWO), Shire, Bayer, 
Pfizer, Agentschap voor Innovatie door Wetenschap en Technologie (IWT), Vrije Universiteit Brussel Industrieel Onderzoeksfonds Groups of Excellence in Applied Research (VUB-IOF-GEAR) (GENEFIX), Strategic Research Program (SRP)Groeier, and the Willy Gepts Fund for providing financial support.

\section{AUTHOR DISCLOSURE}

The authors have patent applications and granted patents in the field of gene therapy for hemophilia. They also received industrial grants and/or consultancies from various companies involved in gene therapy for hemophilia (Shire, Bayer, Pfizer).

\section{REFERENCES}

1. Pierce GF, Lillicrap D, Pipe SW, Vandendriessche T. Gene therapy, bioengineered clotting factors and novel technologies for hemophilia treatment. J Thromb Hemost 2007;5:901-906.

2. Hartmann J, Croteau SE. 2017 Clinical trials update: Innovations in hemophilia therapy. Am J Hematol 2016;91:1252-1260.

3. Lillicrap D, VandenDriessche T, High K. Cellular and genetic therapies for haemophilia. Haemophilia 2006;12 Suppl 3:36-41.

4. Chuah MK, Collen D, VandenDriessche T. Gene therapy for hemophilia. J Gene Med 2001;3: 3-20.

5. Chuah MK, Nair N, VandenDriessche T. Recent progress in gene therapy for hemophilia. Hum Gene Ther 2012;23:557-565.

6. Annoni A, Cantore A, Della Valle P, et al. Liver gene therapy by lentiviral vectors reverses antifactor IX pre-existing immunity in haemophilic mice. EMBO Mol Med 2013;5:1684-1697.

7. Markusic DM, Hoffman BE, Perrin GQ, et al. Effective gene therapy for haemophilic mice with pathogenic factor IX antibodies. EMBO Mol Med 2013;5:1698-1709.

8. Kaufman RJ, Pipe SW, Tagliavacca L, Swaroop M, Moussalli M. Biosynthesis, assembly and secretion of coagulation factor VIII. Blood Coagul Fibrinolysis 1997;8 Suppl 2:S3-14.

9. Chuah MK, VandenDriessche T, Morgan RA. Development and analysis of retroviral vectors expressing human factor VIII as a potential gene therapy for hemophilia A. Hum Gene Ther 1995; 6:1363-1377.

10. Wu Z, Sun J, Zhang T, et al. Optimization of selfcomplementary AAV vectors for liver-directed expression results in sustained correction of hemophilia B at low vector dose. Mol Ther 2008; 16:280-289.

11. Miao $\mathrm{CH}$, Ohashi K, Patijn GA, et al. Inclusion of the hepatic locus control region, an intron, and untranslated region increases and stabilizes hepatic factor IX gene expression in vivo but not in vitro. Mol Ther 2000;1:522-532.

12. Wang L, Takabe K, Bidlingmaier SM, III CR, Verma IM. Sustained correction of bleeding disorder in hemophilia B mice by gene therapy. Proc Natl Acad Sci U S A 1999;96:39063910.
13. Nair N, Rincon MY, Evens H, et al. Computationally designed liver-specific transcriptional modules and hyperactive factor IX improve hepatic gene therapy. Blood 2014; 123:3195-3199.

14. Chuah MK, Petrus I, De Bleser P, et al. Liverspecific transcriptional modules identified by genome-wide in silico analysis enable efficient gene therapy in mice and non-human primates. Mol Ther 2014;22:1605-1613.

15. Di Matteo M, Samara-Kuko E, Ward NJ, et al. Hyperactive piggyBac transposons for sustained and robust liver-targeted gene therapy. Mol Ther 2014;22:1614-1624.

16. Brown BD, Cantore A, Annoni A, et al. A microRNA-regulated lentiviral vector mediates stable correction of hemophilia B mice. Blood 2007;110:4144-4152.

17. Annoni A, Brown BD, Cantore A, Sergi LS, Naldini $L$, Roncarolo MG. In vivo delivery of a microRNA-regulated transgene induces antigenspecific regulatory $T$ cells and promotes immunologic tolerance. Blood 2009;114:5152-5161.

18. Ward NJ, Buckley SM, Waddington SN, et al. Codon optimization of human factor VIII cDNAs leads to high-level expression. Blood 2011;117: 798-807.

19. McIntosh J, Lenting PJ, Rosales C, et al. Therapeutic levels of FVIII following a single peripheral vein administration of rAAV vector encoding a novel human factor VIII variant. Blood 2013;121:3335-3344.

20. Pittman DD, Alderman EM, Tomkinson KN, Wang JH, Giles AR, Kaufman RJ. Biochemical, immunological, and in vivo functional characterization of B-domain-deleted factor VIII. Blood 1993;81:2925-2935.

21. Miao HZ, Sirachainan N, Palmer $L$, et al. Bioengineering of coagulation factor VIII for improved secretion. Blood 2004;103:3412-3419.

22. Siner JI, lacobelli NP, Sabatino DE, et al. Minimal modification in the factor VIII B-domain sequence ameliorates the murine hemophilia $A$ phenotype. Blood 2013;121:4396-4403.

23. Doering CB, Denning G, Dooriss K, et al. Directed engineering of a high-expression chimeric transgene as a strategy for gene therapy of hemophilia A. Mol Ther 2009;17:1145-1154.
24. Lange AM, Altynova ES, Nguyen GN, Sabatino DE. Overexpression of factor VIII after AAV delivery is transiently associated with cellular stress in hemophilia A mice. Mol Ther Methods Clin Dev 2016;3:16064.

25. Chuah MK, Schiedner G, Thorrez L, et al. Therapeutic factor VIII levels and negligible toxicity in mouse and dog models of hemophilia A following gene therapy with high-capacity adenoviral vectors. Blood 2003;101:1734-1743.

26. Cantore A, Nair N, Della Valle $P$, et al. Hyperfunctional coagulation factor IX improves the efficacy of gene therapy in hemophilic mice. Blood 2012;120:4517-4520.

27. Simioni $\mathrm{P}$, Tormene $\mathrm{D}$, Tognin $\mathrm{G}$, et al. X-linked thrombophilia with a mutant factor IX (factor IX Padua). New Engl J Med 2009;361:1671-1675.

28. Finn JD, Nichols TC, Svoronos N, et al. The efficacy and the risk of immunogenicity of FIX Padua (R338L) in hemophilia B dogs treated by AAV muscle gene therapy. Blood 2012;120: 4521-4523.

29. Crudele JM, Finn JD, Siner JI, et al. AAV liver expression of FIX-Padua prevents and eradicates FIX inhibitor without increasing thrombogenicity in hemophilia B dogs and mice. Blood 2015;125: 1553-1561.

30. Monahan PE, Sun J, Gui T, et al. Employing a gain-of-function factor IX variant R338L to advance the efficacy and safety of hemophilia $B$ human gene therapy: Preclinical evaluation supporting an ongoing adeno-associated virus clinical trial. Hum Gene Ther 2015;26:69-81.

31. Schuettrumpf J, Herzog RW, Schlachterman A, Kaufhold A, Stafford DW, Arruda VR. Factor IX variants improve gene therapy efficacy for hemophilia B. Blood 2005;105:2316-2323.

32. Kren BT, Unger GM, Sjeklocha L, et al. Nanocapsule-delivered Sleeping Beauty mediates therapeutic Factor VIII expression in liver sinusoidal endothelial cells of hemophilia A mice. J Clin Invest 2009;119:2086-2099.

33. Mingozzi F, Liu YL, Dobrzynski E, et al. Induction of immune tolerance to coagulation factor IX antigen by in vivo hepatic gene transfer. $\mathrm{J}$ Clin Invest 2003;111:1347-1356.

34. Matsui H, Shibata M, Brown B, et al. A murine model for induction of long-term immunologic 
tolerance to factor VIII does not require persistent detectable levels of plasma factor VIII and involves contributions from Foxp3+ $T$ regulatory cells. Blood 2009;114:677-685.

35. Cao 0, Loduca PA, Herzog RW. Role of regulatory $T$ cells in tolerance to coagulation factors. $J$ Thromb Hemost 2009;7 Suppl 1:88-91.

36. Wang L, Cao O, Swalm B, Dobrzynski E, Mingozzi F, Herzog RW. Major role of local immune responses in antibody formation to factor IX in AAV gene transfer. Gene Ther 2005;12:14531464.

37. Buchlis G, Podsakoff GM, Radu A, et al. Factor IX expression in skeletal muscle of a severe hemophilia B patient 10 years after AAV-mediated gene transfer. Blood 2012;119:3038-3041.

38. Montgomery RR, Shi 0. Platelet and endothelial expression of clotting factors for the treatment of hemophilia. Thromb Res 2012;129:S46-48.

39. Lin Y, Chang L, Solovey A, Healey JF, Lollar P, Hebbel RP. Use of blood outgrowth endothelia cells for gene therapy for hemophilia A. Blood 2002:99:457-462.

40. Roth DA, Tawa NE, Jr., O'Brien JM, Treco DA, Selden RF, Factor VTTSG. Nonviral transfer of the gene encoding coagulation factor VIII in patients with severe hemophilia A. New Engl J Med 2001;344:1735-1742.

41. Yant SR, Meuse L, Chiu W, Ivics Z, Izsvak Z, Kay MA. Somatic integration and long-term transgene expression in normal and haemophilic mice using a DNA transposon system. Nat Genet 2000;25:35-41.

42. Mates L, Chuah MK, Belay E, et al. Molecular evolution of a novel hyperactive Sleeping Beauty transposase enables robust stable gene transfer in vertebrates. Nat Genet 2009;41:753-761.

43. VandenDriessche T, Ivics Z, Izsvak Z, Chuah MK. Emerging potential of transposons for gene therapy and generation of induced pluripotent stem cells. Blood 2009;114:1461-1468.

44. VandenDriessche T, Vanslembrouck V, Goovaerts I, et al. Long-term expression of human coagulation factor VIII and correction of hemophilia A after in vivo retroviral gene transfer in factor VIII-deficient mice. Proc Natl Acad Sci U S A 1999;96:10379-10384

45. Xu L, Gao C, Sands MS, et al. Neonatal or hepatocyte growth factor-potentiated adult gene therapy with a retroviral vector results in therapeutic levels of canine factor IX for hemophilia B. Blood 2003;101:3924-3932.

46. Xu L, Nichols TC, Sarkar R, McCorquodale S Bellinger DA, Ponder KP. Absence of a desmopressin response after therapeutic expression of factor VIII in hemophilia A dogs with liverdirected neonatal gene therapy. Proc Natl Acad Sci U S A 2005;102:6080-6085.

47. Powell JS, Ragni MV, White GC, 2nd, et al Phase 1 trial of FVIII gene transfer for severe hemophilia A using a retroviral construct ad- ministered by peripheral intravenous infusion Blood 2003:102:2038-2045.

48. VandenDriessche T. Challenges and progress in gene therapy for hemophilia A. Blood 2003;102: 1938-1939.

49. Matrai J, Chuah MK, VandenDriessche T. Preclinical and clinical progress in hemophilia gene therapy. Curr Opin Hematol 2010;17:387-392.

50. Matrai J, Chuah MK, VandenDriessche T. Recent advances in lentiviral vector development and applications. Mol Ther 2010:18:477-490.

51. VandenDriessche T, Thorrez L, Naldini L, et al Lentiviral vectors containing the human immunodeficiency virus type-1 central polypurine tract can efficiently transduce nondividing hepatocytes and antigen-presenting cells in vivo. Blood 2002:100:813-822.

52. Follenzi A, Battaglia M, Lombardo A, Annoni $A$ Roncarolo MG, Naldini L. Targeting lentiviral vector expression to hepatocytes limits transgene-specific immune response and establishes long-term expression of human antihemophilic factor IX in mice. Blood 2004;103:37003709

53. Vandendriessche T, Thorrez L, Acosta-Sanchez A et al. Efficacy and safety of adeno-associated viral vectors based on serotype 8 and 9 vs. lentiviral vectors for hemophilia B gene therapy. J Thromb Hemost 2007:5:16-24.

54. Merlin S, Cannizzo ES, Borroni E, et al. A Nove platform for immune tolerance induction in hemophilia A mice. Mol Ther 2017;25:1815-1830.

55. Matrai J, Cantore A, Bartholomae CC, et al Hepatocyte-targeted expression by integrasedefective lentiviral vectors induces antigenspecific tolerance in mice with low genotoxic risk. Hepatology 2011;53:1696-1707.

56. Cantore A, Ranzani M, Bartholomae CC, et al Liver-directed lentiviral gene therapy in a dog model of hemophilia B. Sci Transl Med 2015;7: 277ra228

57. Vandendriessche T, Chuah MK. Clinical progress in gene therapy: sustained partial correction of the bleeding disorder in patients suffering from severe hemophilia B. Hum Gene Ther 2012;23 4-6.

58. Shi 0, Wilcox DA, Fahs SA, et al. Lentivirusmediated platelet-derived factor VIII gene therapy in murine haemophilia $\mathrm{A}$. $\mathrm{J}$ Thromb Hemost 2007:5:352-361.

59. Du LM, Nurden P, Nurden AT, et al. Platelettargeted gene therapy with human factor VIII establishes haemostasis in dogs with haemophilia A. Nat Commun 2013:4:2773

60. Kuether EL, Schroeder JA, Fahs SA, et al. Lentivirus-mediated platelet gene therapy of murine hemophilia A with pre-existing antifactor VIII immunity. J Thromb Hemost 2012;10: 1570-1580.

61. Shi Q, Schroeder JA, Kuether EL, Montgomery RR. The important role of von Willebrand factor in platelet-derived FVIII gene therapy for murine hemophilia $A$ in the presence of inhibitory antibodies. J Thromb Hemost 2015;13:1301-1309.

62. Wang $X$, Shin SC, Chiang AF, et al. Intraosseous delivery of lentiviral vectors targeting factor VIII expression in platelets corrects murine hemophilia A. Mol Ther 2015;23:617-626.

63. Levy C, Fusil F, Amirache F, et al. Baboon envelope pseudotyped lentiviral vectors efficiently transduce human $B$ cells and allow active factor IX B cell secretion in vivo in NOD/ SCIDgammac-/- mice. J Thromb Hemost 2016; 14:2478-2492

64. Montini E, Cesana D, Schmidt M, et al. The genotoxic potential of retroviral vectors is strongly modulated by vector design and integration site selection in a mouse model of HSC gene therapy. J Clin Invest 2009;119:964-975.

65. Ehrhardt A, Kay MA. A new adenoviral helperdependent vector results in long-term therapeutic levels of human coagulation factor IX at low doses in vivo. Blood 2002;99:39233930.

66. Brown BD, Shi CX, Powell S, Hurlbut D, Graham FL, Lillicrap D. Helper-dependent adenoviral vectors mediate therapeutic factor VIII expression for several months with minimal accompanying toxicity in a canine model of severe hemophilia A. Blood 2004;103:804-810.

67. Brunetti-Pierri N, Stapleton GE, Law M, et al Efficient, long-term hepatic gene transfer using clinically relevant HDAd doses by balloon occlusion catheter delivery in nonhuman primates. Mol Ther 2009:17:327-333.

68. Herzog RW, Yang EY, Couto LB, et al. Long-term correction of canine hemophilia $\mathrm{B}$ by gene transfer of blood coagulation factor IX mediated by adeno-associated viral vector. Nat Med 1999; 5:56-63.

69. Herzog RW, Hagstrom JN, Kung SH, et al. Stable gene transfer and expression of human blood coagulation factor IX after intramuscular injection of recombinant adeno-associated virus. Proc Natl Acad Sci U S A 1997;94:5804-5809.

70. Herzog RW, Mount JD, Arruda VR, High KA Lothrop CD, Jr. Muscle-directed gene transfer and transient immune suppression result in sustained partial correction of canine hemophilia B caused by a null mutation. Mol Ther 2001;4: 192-200.

71. Arruda VR, Stedman HH, Haurigot V, et al. Peripheral transvenular delivery of adenoassociated viral vectors to skeletal muscle as a novel therapy for hemophilia B. Blood 2010;115 4678-4688.

72. Wang L, Herzog RW. AAV-mediated gene transfer for treatment of hemophilia. Curr Gene Ther 2005;5:349-360.

73. Kay MA, Manno CS, Ragni MV, et al. Evidence for gene transfer and expression of factor IX in haemophilia B patients treated with an AAV vector. Nat Genet 2000;24:257-261. 
74. Grimm D, Lee JS, Wang L, et al. In vitro and in vivo gene therapy vector evolution via multispecies interbreeding and retargeting of adenoassociated viruses. J Virol 2008;82:5887-5911.

75. Lisowski L, Dane AP, Chu K, et al. Selection and evaluation of clinically relevant AAV variants in a xenograft liver model. Nature 2014;506:382386.

76. Zhong L, Li B, Mah CS, et al. Next generation of adeno-associated virus 2 vectors: point mutations in tyrosines lead to high-efficiency transduction at lower doses. Proc Natl Acad Sci U S A 2008; 105:7827-7832.

77. Snyder RO, Miao CH, Patijn GA, et al. Persistent and therapeutic concentrations of human factor IX in mice after hepatic gene transfer of recombinant AAV vectors. Nat Genet 1997;16: 270-276.

78. Snyder RO, Miao C, Meuse L, et al. Correction of hemophilia B in canine and murine models using recombinant adeno-associated viral vectors. Nat Med 1999;5:64-70.

79. Wang L, Nichols TC, Read MS, Bellinger DA, Verma IM. Sustained expression of therapeutic level of factor IX in hemophilia B dogs by AAVmediated gene therapy in liver. Mol Ther 2000;1: 154-158.

80. Mount JD, Herzog RW, Tillson DM, et al. Sustained phenotypic correction of hemophilia B dogs with a factor IX null mutation by liver-directed gene therapy. Blood 2002;99:2670-2676.

81. Nathwani AC, Davidoff AM, Hanawa $H$, et al. Sustained high-level expression of human factor IX (hFIX) after liver-targeted delivery of recombinant adeno-associated virus encoding the $\mathrm{hFIX}$ gene in rhesus macaques. Blood 2002;100:16621669.

82. Nathwani AC, Gray JT, Ng CY, et al. Selfcomplementary adeno-associated virus vectors containing a novel liver-specific human factor IX expression cassette enable highly efficient transduction of murine and nonhuman primate liver. Blood 2006;107:2653-2661.

83. Dobrzynski E, Fitzgerald JC, Cao O, Mingozzi F, Wang L, Herzog RW. Prevention of cytotoxic T lymphocyte responses to factor IX-expressing hepatocytes by gene transfer-induced regulatory T cells. Proc Natl Acad Sci U S A 2006;103:45924597.

84. Manno CS, Pierce GF, Arruda VR, et al. Successful transduction of liver in hemophilia by AAV-Factor IX and limitations imposed by the host immune response. Nat Med 2006;12: 342-347.

85. Mingozzi F, Maus MV, Hui DJ, et al. CD8(+) Tcell responses to adeno-associated virus capsid in humans. Nat Med 2007;13:419-422.

86. Pien GC, Basner-Tschakarjan E, Hui DJ, et al. Capsid antigen presentation flags human hepa- tocytes for destruction after transduction by adeno-associated viral vectors. J Clin Invest 2009;119:1688-1695.

87. Martino AT, Basner-Tschakarjan E, Markusic $D M$, et al. Engineered AAV vector minimizes in vivo targeting of transduced hepatocytes by capsid-specific CD8+ T cells. Blood 2013;121: 2224-2233.

88. Nathwani AC, Tuddenham EG, Rangarajan S, et al. Adenovirus-associated virus vectormediated gene transfer in hemophilia B. New Engl J Med 2011;365:2357-2365.

89. Gao GP, Alvira MR, Wang L, Calcedo R, Johnston J, Wilson JM. Novel adeno-associated viruses from rhesus monkeys as vectors for human gene therapy. Proc Natl Acad Sci U S A 2002;99:11854-11859.

90. Gao G, Vandenberghe LH, Alvira MR, et al. Clades of Adeno-associated viruses are widely disseminated in human tissues. J Virol 2004;78: 6381-6388.

91. Vandenberghe LH, Wang L, Somanathan S, et al. Heparin binding directs activation of $T$ cells against adeno-associated virus serotype 2 capsid. Nat Med 2006;12:967-971.

92. McCarty DM, Monahan PE, Samulski RJ. Selfcomplementary recombinant adeno-associated virus (scAAV) vectors promote efficient transduction independently of DNA synthesis. Gene Ther 2001;8:1248-1254.

93. Sarkar R, Xiao W, Kazazian HH, Jr. A single adeno-associated virus (AAV)-murine factor VIII vector partially corrects the hemophilia A phenotype. J Thromb Hemost 2003;1:220-226.

94. Sarkar R, Tetreault R, Gao G, et al. Total correction of hemophilia A mice with canine FVIII using an AAV 8 serotype. Blood 2004;103:12531260.

95. Scallan CD, Lillicrap D, Jiang H, et al. Sustained phenotypic correction of canine hemophilia $A$ using an adeno-associated viral vector. Blood 2003;102:2031-2037.

96. Sabatino DE, Lange AM, Altynova ES, et al. Efficacy and safety of long-term prophylaxis in severe hemophilia A dogs following liver gene therapy using AAV vectors. Mol Ther 2011;19: 442-449.

97. Jiang H, Lillicrap D, Patarroyo-White $S$, et al. Multiyear therapeutic benefit of AAV serotypes 2, 6, and 8 delivering factor VIII to hemophilia A mice and dogs. Blood 2006;108: 107-115.

98. Nguyen GN, George LA, Siner Jl, et al. Novel factor VIII variants with a modified furin cleavage site improve the efficacy of gene therapy for hemophilia A. J Thromb Hemost 2017;15: 110-121.

99. Brown HC, Wright JF, Zhou S, et al. Bioengineered coagulation factor VIII enables long-term correction of murine hemophilia A following liver-directed adeno-associated viral vector delivery. Mol Ther Methods Clin Dev 2014;1: 14036.

100. Donsante A, Miller DG, Li Y, et al. AAV vector integration sites in mouse hepatocellular carcinoma. Science 2007;317:477.

101. Li H, Malani N, Hamilton SR, et al. Assessing the potential for AAV vector genotoxicity in a murine model. Blood 2011;117:3311-3319.

102. Nault JC, Datta S, Imbeaud S, et al. Recurrent AAV2-related insertional mutagenesis in human hepatocellular carcinomas. Nat Genet 2015;47: 1187-1193.

103. Berns Kl, Byrne BJ, Flotte TR, et al. AdenoAssociated Virus Type 2 and Hepatocellular Carcinoma? Hum Gene Ther 2015;26:779-781.

104. Buning H, Schmidt M. Adeno-associated Vector Toxicity-To Be or Not to Be? Mol Ther 2015;23: 1673-1675.

105. Chandler RJ, LaFave MC, Varshney GK, et al. Vector design influences hepatic genotoxicity after adeno-associated virus gene therapy. J Clin Invest 2015;125:870-880.

106. Chandler RJ, Sands MS, Venditti CP. Recombinant Adeno-Associated Viral Integration and Genotoxicity: Insights from Animal Models. Hum Gene Ther 2017;28:314-322.

107. Logan GJ, Dane AP, Hallwirth CV, et al. Identification of liver-specific enhancer-promoter activity in the $3^{\prime}$ untranslated region of the wildtype AAV2 genome. Nat Genet 2017;49:12671273.

108. Li H, Haurigot $V$, Doyon $Y$, et al. In vivo genome editing restores haemostasis in a mouse model of haemophilia. Nature 2011:475:217221.

109. Anguela XM, Sharma R, Doyon Y, et al. Robust ZFN-mediated genome editing in adult hemophilic mice. Blood 2013;122:3283-3287.

110. Sharma $R$, Anguela $X M$, Doyon $Y$, et al. In vivo genome editing of the albumin locus as a platform for protein replacement therapy. Blood 2015;126:1777-1784

111. Park CY, Kim DH, Son JS, et al. Functional Correction of Large Factor VIII Gene Chromosomal Inversions in Hemophilia A Patient-Derived iPSCs Using CRISPR-Cas9. Cell Stem Cell 2015;17: 213-220.

112. Barzel A, Paulk NK, Shi Y, et al., Promoterless gene targeting without nucleases ameliorates haemophilia B in mice. Nature 2015; 517:360364.

Received for publication June 28, 2017; accepted after revision July 28, 2017.

Published online: August 3, 2017. 